\title{
Author Correction: Is body-weight supported treadmill training or robotic-assisted gait training superior to overground gait training and other forms of physiotherapy in people with spinal cord injury? A systematic review
}

\section{J. Mehrholz ${ }^{1,2} \cdot$ L. A. Harvey ${ }^{3}$ S. Thomas ${ }^{1} \cdot$ B. Elsner $^{2}$}

Published online: 24 January 2018

(c) International Spinal Cord Society 2018

Correction to: Spinal Cord advance online publication 11 April 2017; https://doi.org/10.1038/sc.2017.31.

Since publication of this paper, the authors have become aware of two minor errors in the labelling of Figures 4 and 5. They are:

1. Figure 4: the title of column 2 is incorrect. It should read "Robotic" (not BWSTT).

The original article can be found online at https://doi.org/10.1038/sc. 2017.31.

J. Mehrholz

jan.mehrholz@klinik-bavaria.de

1 Institute of Science, Private European Medical Academy of the Klinik Bavaria Kreischa, Kreischa, Germany
2. Figure 5: the titles of columns 2 and 3 are the wrong way around. So column 2 should be "Robotic" (not "Overground") and column 3 should read "Overground" (not "Robotic").

The authors apologise for these errors and any inconvenience caused.
2 Department of Public Health, Dresden Medical School, Technical University Dresden, Dresden, Germany

3 John Walsh Centre for Rehabilitation Research, Kolling Institute, Sydney Medical School/Northern, University of Sydney, Sydney, New South Wales, Australia 